\title{
Assessment of Refractive Outcome of Photo Refractive Keratectomy in Myopia and Myopic Astigmatism
}

\author{
Aisha Fawad ${ }^{1}$, Sadia Humayun², Mazhar Ishaq ${ }^{3}$, Sabahat Arzoo², Syed Fawad Mashhadi and Quratulain \\ Humayun $^{5}$ \\ ${ }^{1}$ Department of Eye, Combined Military Hospital, Armed Forces Post-Graduate Medical Institute, Nowshera, Pakistan \\ ${ }^{2}$ Refractive Surgical Department, Armed Forces Institute of Ophthalmology, National University of Medical Sciences, Rawalpindi, \\ Pakistan \\ ${ }^{3}$ Department of Eye, The Eye Consultants, Rawalpindi, Pakistan \\ ${ }^{4}$ Department of Community Medicine, Army Medical College, Rawalpindi, Pakistan \\ ${ }^{5}$ Child and Family Asthma Studies Centre, Women and Children's Hospital of Buffalo, New York, United States of America
}

\begin{abstract}
Objectives: To determine the refractive outcomes of wavefront optimized photo refractive keratectomy (PRK) in patients having myopia and myopic astigmatism in terms of efficacy, safety, accuracy, stability and predictability of the procedure.

Study Design: Quasi-experimental study.

Place and Duration of Study: Refractive Surgical Unit, Armed Forces Institute of Ophthalmology, National University of Medical Sciences, Rawalpindi, Pakistan, from October 2013 to August 2018.

Methodology: Patients having myopia and myopic astigmatism, consented to be a part of the study, underwent meticulous screening consisting of detailed medical history, ocular examination and investigations. Each case was compiled and evaluated. Those who satisfied the criteria for refractive surgery were subjected to PRK procedure. Postoperative follow-up was carried out and parameters were recorded at designated intervals of 1 week, 1, 3, 6 and 12 months.

Results: This study included 208 eyes of 106 patients with mean age of $25.33 \pm 5.196$ years. At the end of one year, $97 \%$ (202 out of 208) eyes had uncorrected distance visual acuity (UDVA) better than 20/25 with efficacy index of $1.009 \pm 0.132$ and safety index of $1.019 \pm 0.126$. None of the eyes lost two or more lines. In this study, 207 out of 208 eyes remained within \pm 0.50 diopter (D) of intended spherical equivalent (SEQ). Results showed $100 \%$ accuracy of procedure since all the study patients achieved SEQ within $\pm 1 \mathrm{D}$.
\end{abstract}

Conclusion: PRK is an effective, predictable, and safe treatment procedure for correcting myopia and myopic astigmatism.

Key Words: Photorefractive keratectomy, Myopia, Refractive surgery, Myopic astigmatism, Uncorrected distance visual acuity.

How to cite this article: Fawad A, Humayun S, Ishaq M, Arzoo S, Mashhadi SF, Humayun Q. Assessment of Refractive Outcome of Photo Refractive Keratectomy in Myopia and Myopic Astigmatism. J Coll Physicians Surg Pak 2022; 32(03):329-334.

\section{INTRODUCTION}

Photorefractive keratectomy (PRK) is a corneal surface ablation technique in which corneal epithelium is removed and stroma is ablated with the help of an excimer laser to achieve desired refractive correction. PRK was first developed by Dr. Steven Trokel, first performed by Dr. Theo Sieler in 1987, and approved by FDAin $1996 .{ }^{1}$

Correspondence to: Dr. Sadia Humayun, Refractive Surgical Department, Armed Forces Institute of Ophthalmology (AFIO), National University of Medical Sciences, Rawalpindi, Pakistan

E-mail: sadia.humayun@yahoo.com

Received: May 03, 2021; Revised: January 03, 2022;

Accepted: January 04, 2022

DOI: https://doi.org/10.29271/jcpsp.2022.03.329
Epithelium can be removed manually with a blunt spatula or brush. Its removal can also be alcohol assisted or with the help of excimer laser; in which case, it is termed as transepithelial PRK. ${ }^{2,3}$ Growth of epithelium takes days and it can be associated with significant pain during early postoperative period and a relatively longer visual recovery time when compared to LASIK. ${ }^{4}$ Healing can be associated with corneal haze, which may or may not improve with time. ${ }^{5}$ Despite all this, the refractive scientists and surgeons value this procedure for its better safety profile in relatively thinnercorneas, atypical corneas; and in certain situations where flap cannot be created or flap related complications have to be avoided. ${ }^{6,7}$ In developing countries like Pakistan, it is an affordable alternate to LASIK. ${ }^{8}$

The authors aim to begin their series of research on PRK and related aspects, with projecting and analysing its basic refractive outcomes in myopia and myopic astigmatism as per the standard graphs of refractive surgery ${ }^{9}$ in the local population; which to the best of the authors' knowledge, has not been published so far. 


\begin{tabular}{|c|c|c|c|c|c|c|c|c|}
\hline \multirow[b]{2}{*}{ Variable } & \multirow{2}{*}{$\begin{array}{l}\text { Pre-operative } \\
\text { mean }(M \pm S D)\end{array}$} & \multicolumn{3}{|c|}{ Post-operative mean $(\mathrm{M} \pm \mathrm{SD})$} & \multicolumn{4}{|c|}{ p-value (two-tail) } \\
\hline & & 1 month & 6 months & 12 months & $\begin{array}{l}\text { Pre-op vs } 1 \\
\text { month }\end{array}$ & $\begin{array}{l}\text { Pre-op vs } 6 \\
\text { month }\end{array}$ & $\begin{array}{l}\text { Pre-op vs } \\
12 \text { month }\end{array}$ & $\begin{array}{l}6 \text { months vs } \\
12 \text { months }\end{array}$ \\
\hline $\begin{array}{l}\text { UDVA } \\
\text { (LogMAR) }\end{array}$ & $1.114 \pm 0.306$ & $\begin{array}{l}0.084 \pm \\
0.099\end{array}$ & $\begin{array}{l}0.020 \pm \\
0.054\end{array}$ & $\begin{array}{l}0.016 \pm \\
0.047\end{array}$ & $<0.001^{*}$ & $<0.001^{*}$ & $<0.001^{*}$ & $0.009 *$ \\
\hline SEQ (D) & $-3.536 \pm 1.451$ & $\begin{array}{l}-0.173 \pm \\
0.264\end{array}$ & $\begin{array}{l}-0.022 \pm \\
0.168\end{array}$ & $\begin{array}{l}0.029 \pm \\
0.099\end{array}$ & $<0.001^{*}$ & $<0.001^{*}$ & $<0.001^{*}$ & 0.596 \\
\hline $\begin{array}{l}\text { CDVA } \\
\text { (LogMAR) }\end{array}$ & $0.016 \pm 0.056$ & $\begin{array}{l}0.049 \pm \\
0.064\end{array}$ & $\begin{array}{l}0.013 \pm \\
0.044\end{array}$ & $\begin{array}{l}0.010 \pm \\
0.041\end{array}$ & $<0.001^{*}$ & 0.215 & 0.047 & 0.110 \\
\hline
\end{tabular}

\section{METHODOLOGY}

This one-sample pretest-posttest quasi experimental study was approved by the Hospital Review Board and Ethical Committee. It included 208 eyes of 106 patients from October 2013 to August 2018 at Refractive Surgical Unit, Armed Forces Institute of Ophthalmology, Rawalpindi. Patients who consented to be a part of the study were recruited through purposive sampling and were treated by two surgeons, who had identical techniques and protocols. Patients were of 18 years and above and had myopia or myopic astigmatism of upto $6 \mathrm{D}$. Those with estimated postoperative flat keratometry $(K)$ of more than $34 \mathrm{D}$ and estimated residual stromal bed of more than 300 microns were included in the study. However, those having unstable refraction, pregnancy and lactation, systemic diseases (like collagen vascular disorders, uncontrolled diabetes mellitus, autoimmune disorders, and immune deficiency disorders), using drugs like isotretinoins, antidepressants, amiodarone, or ocular pathologies, were excluded. Contact lens users were assessed and included after two weeks of discontinuation.

Preoperative evaluation included detailed history, visual acuity assessment, uncorrected distance visual acuity (UDCA), corrected distance visual acuity (CDVA), VA with spectacles, and refraction under cycloplegia, anterior and posterior segment examination with slit lamp microscope, measurement of intra-ocular pressure and tear film breakup time for dry eye assessment. After the examination, patients' tomography, topography, pupillometry and aberrometry were recorded. Topography and pupillometry were carried out with Allegro Topolyzer Vario, tomography of cornea with Wavelight Oculyzer II machine and aberrometry with Wavelight Analyser II (Wavelight GmbH Erlangen, Germany). In each case, the relevant information was organized and evaluated to decide if the patient was an appropriate candidate for the refractive surgery procedure. Once it was established, patients' detailed counsellings were performed both by the counsellors as well as the surgeons. Procedure options and choices were offered and their details were discussed, possible risks and benefits, risk of retreatment, presbyopia and postoperative care were explained to the patients. Reading material and videos of the procedure were shown to the patients. Those who consented for photorefractive keratectomy (PRK) or those for whom this procedure was considered the first choice of treatment by the surgeons, were registered in this study. Finally, written informed consent was sought from each participant prior to the procedure.

On the day of surgery, refraction was repeated to confirm the final readings. Target refraction was kept at emmetropia; and data was fed into the planner of EX 500, $1050 \mathrm{~Hz}$ Excimer laser (Wavelight GmbH Erlangen, Germany) and wavefront optimized ablation profile was planned for all the patients. Optical zone, ablation zone and minimum residual stromal bed thickness were kept at 6-6.5 $\mathrm{mm}, 9 \mathrm{~mm}$ and 300 microns, respectively.

Standard protocol for the procedure was adopted. After appropriately positioning the patient, topical anesthesia of $0.5 \%$ proparacaine hydrochloride was instilled, speculum applied and disinfection followed by thorough irrigation of conjunctival sac was carried out. Epithelium was scraped manually with a blunt spatula. The patients were asked to fixate on a target spot, centration was done on cornea, pupil tracking system was activated and laser applied. The stromal bed was washed with cold balanced salt solution and soft bandage contact lens was applied. Speculum was removed and contact lens checked at slit lamp after half an hour. The patients were briefed about the use of drops and what to expect in postoperative period. The prescription included $0.3 \%$ tobramycin and $0.1 \%$ dexamethasone combination drops 4-hourly, and lubricant eye drops every two hours. After three weeks, tobramycin and dexamethasone combination was replaced with $0.1 \%$ fluorometholone eye drops thrice a day for a minimum duration of one month and then tapered according to the individual response of the patients. Patients' follow-ups were performed at the first day, 1 week, 1, 3, 6 and 12 months. The contact lens was removed on the $6^{\text {th }}$ day when the epithelial defect had completely healed.

Calculation of sample size and statistical analysis. Formula used for the calculation of sample size was:

$n=Z 1-\propto 2 \times S D d 2$

In the formula above, Z $1-\alpha / 2$ is the standard normal coefficient at $95 \%$ confidence interval (1.96), SD is the standard deviation and $d$, the absolute error or precision level ( 1 $\mu \mathrm{m})$. Two studies from the available literature were reviewed. Parameters (mean values) reported in these studies were used to calculate sample size. The largest sample size calculated for both the studies was 188; and after adjustment for possibility of attrition it was calculated to be 208 . 


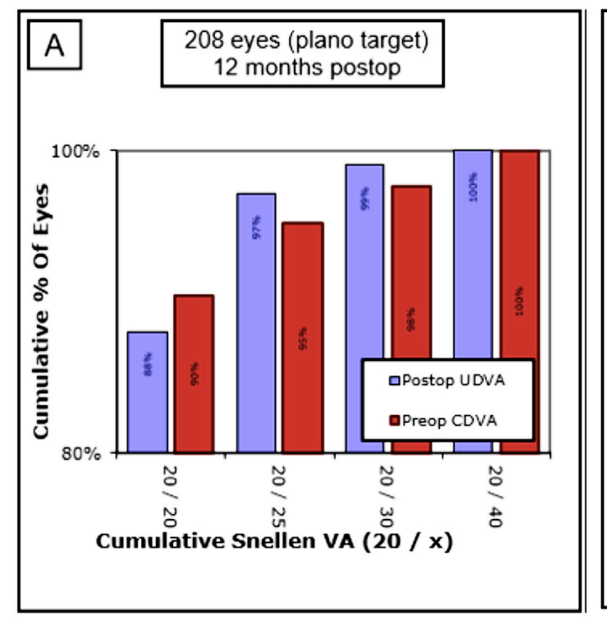

Uncorrected Distance Visual Acuity

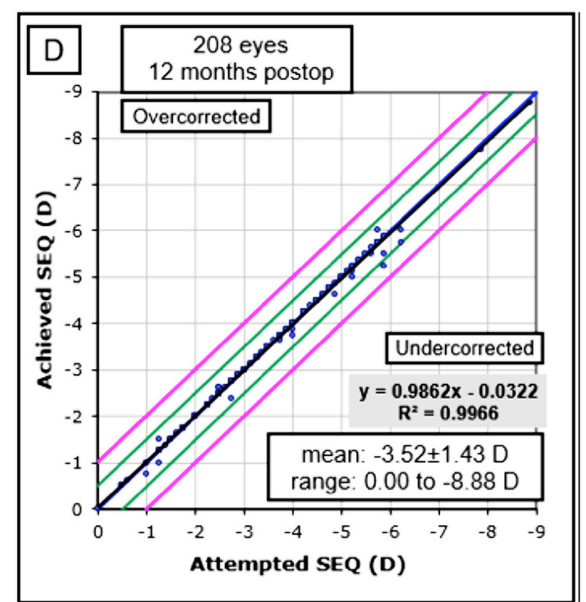

Spherical Equivalent Refraction Attempted vs Achieved

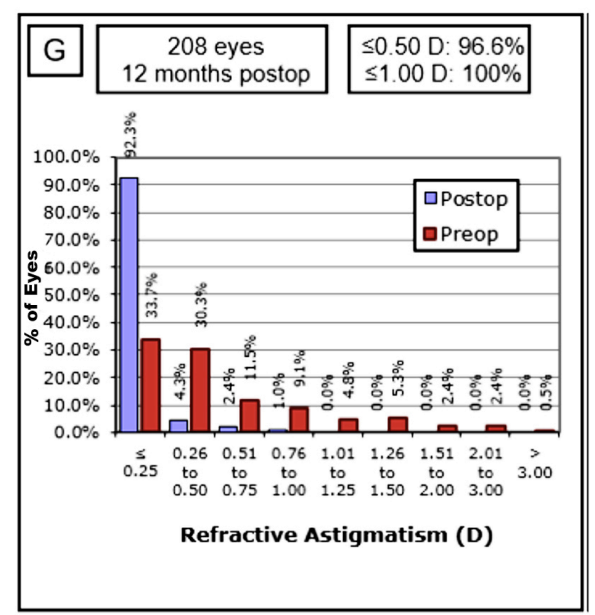

Refractive Astigmatism

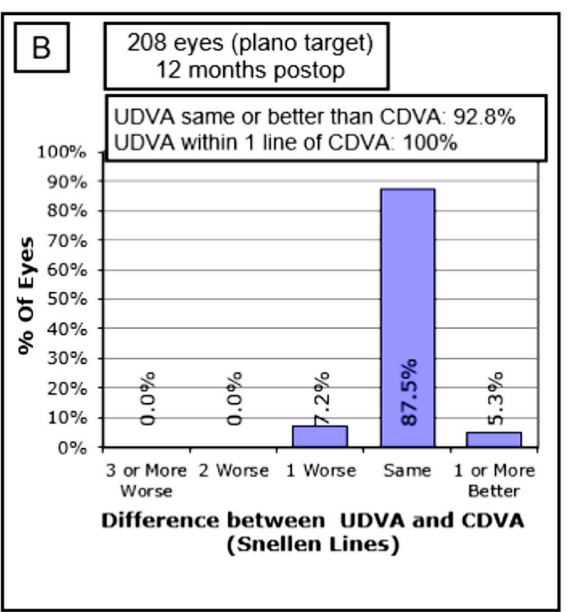

Uncorrected Distance Visual Acuity vs. Corrected Distance Visual Acuity

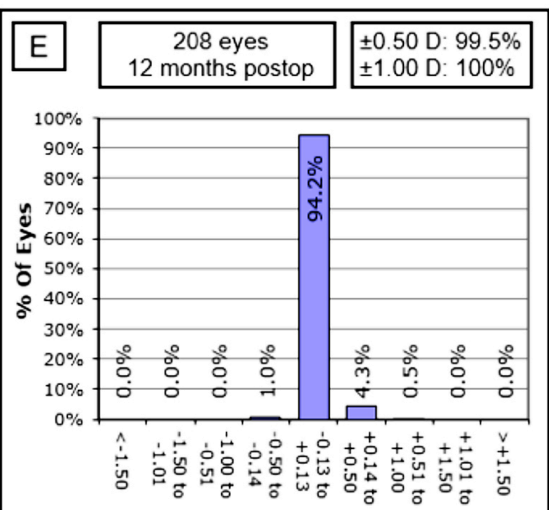

Accuracy of SEQ to Intended Target (D)

\section{Spherical Equivalent Refraction Accuracy}

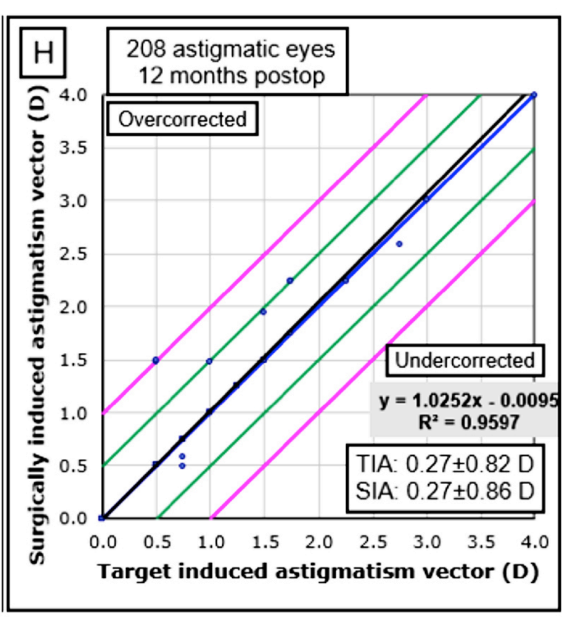

Target Induced Astigmatism vs Surgically Induced Astigmatism

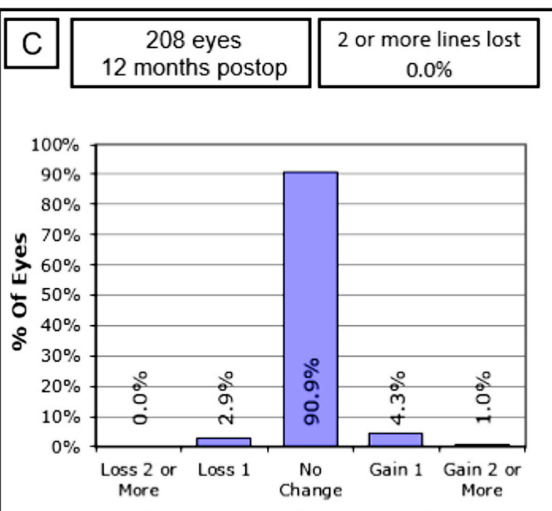

Change in Snellen Lines of CDVA

\section{Change in Corrected Distance Visual} Acuity

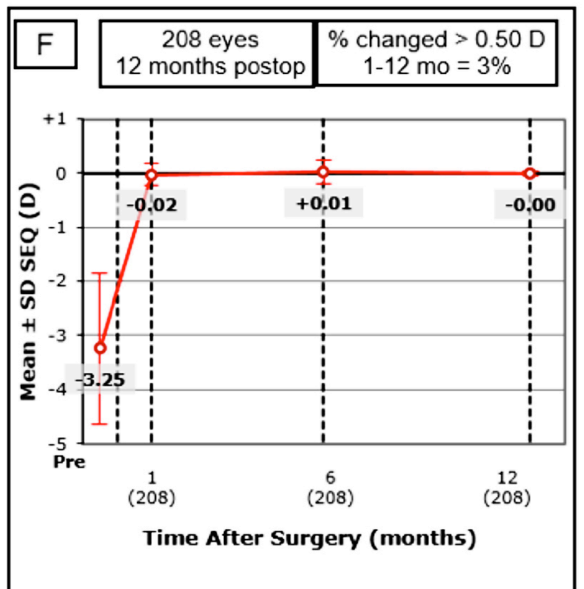

\section{Spherical Equivalent Refraction Stability}

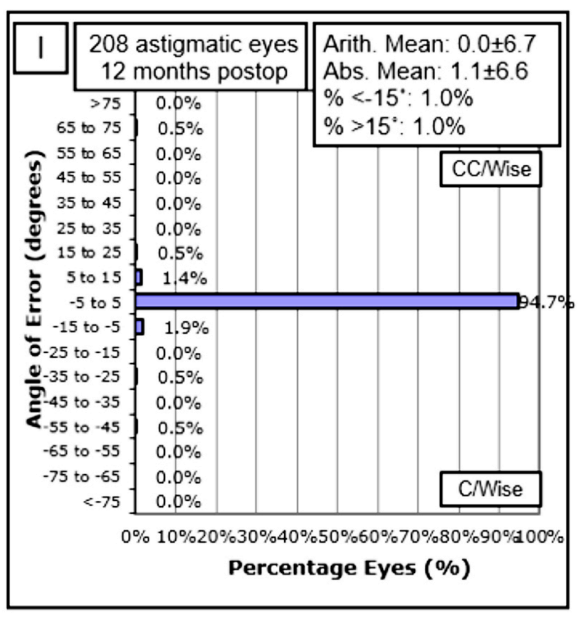

\section{Refractive Astigmatism Angle of} Error

Figure 1: Graphs for refractive surgery outcomes. A: Efficacy profile (comparing postoperative UDVA with preoperative CDVA). B: Difference in terms of loss or gain of Snellen lines between postoperative UDVA and preoperative CDVA. C: Safety profile (change observed in CDVA in terms of loss or gain of Snellen lines). D: Predictability profile (intended SEQ vs. achieved SEQ). E: Accuracy profile (percentage of patients within \pm 1D range of postoperative SEQ). F: Stability profile (changes observed in mean SEQ over time). G: Refractive astigmatism (percentage of patients having postoperative astigmatism within \pm ID range). $\mathrm{H}$ : TIA compared to SIA. I: Refractive astigmatism angle of error between TIA and SIA 
SPSS version 25.0 was used for statistical analysis. Kolmogorov-Smirnov test was applied to test the data for normality. The quantitative data was evaluated by taking mean and standard deviation values. The frequency and percentages were calculated for qualitative data. The paired sample t-test was used for comparison between pre- and post-operative values. Whereas, $p<0.05$ was taken to be significant. The standard graphs used for reporting of refractive surgery outcomes were made to demonstrate the results of the procedure carried out in the study. The analysis of target-induced astigmatism versus surgically-induced astigmatism and attempted SEQ versus achieved SEQ were reported by using linear regression.

\section{RESULTS}

A total of 208 eyes of 106 patients were enrolled in the study. Among these, 97 eyes (46.63\%) were of male patients; whereas, 111 eyes (53.37\%) were of female patients. The mean age of the participants was $25.33 \pm 5.196$ years.

Patients' pre- and post-operative UDVA, spherical equivalent SEQ, value of which is calculated by the algebraic sum of spherical and half of cylindrical power, and CDVA were noted as shown in Table I. The UDVA preoperative and postoperative difference was significant when measured at 1, 6 and 12 months ( $p<0.05)$. The difference was also found to be significant when compared between 6 months and 12 months, in order to look for changes during postoperative time duration $(p<0.05)$. Similarly, for SEQ, there was a significant difference between preoperative and postoperative values when measured at 1,6 and 12 months ( $p<0.05$ ). However, for CDVA, preoperative and postoperative difference was significant at 1 and 12 months and insignificant at 6 months.

Other refractive outcomes included efficacy profile, safety profile, predictability, stability, astigmatism, and comparison of target- induced and surgically-induced astigmatism.

Efficacy is the percentage of the eyes that achieve postoperative uncorrected vision (UDVA) equal to or better than preoperative corrected distance vision (CDVA). In this study at end of 12 months, 99\% (206 from a total of 208) study participants had UDVA of 20/30 or better (Figure 1A). Around 97\% (202) patients experienced UDVA better than 20/25. The postoperative UDVA was compared to preoperative CDVA at 12 months by noting the loss or gain in lines (Figure $1 B$ ), and it was found that $92.8 \%$ (193) participants achieved post-operative UDVA, which was either same or better than preoperative CDVA. Rest of the 7.2\% patients also had UDVA within one line of preoperative CDVA. Efficacy index (i.e., the ratio of the mean postoperative UDVA to the mean preoperative CDVA) was $1.009 \pm 0.132$.

The safety profile of the procedure at 12 months was assessed by comparing preoperative CDVA with postoperative CDVA in terms of loss or gain of Snellen lines (Figure 1C). Out of 208 patients, $90.9 \%$ (189) showed the same and
$5.3 \%$ (11) showed better postoperative CDVA when compared with preoperative value. However, $2.9 \%$ (6) patients experienced loss of one line. None of the patients lost two or more lines. The safety index calculated in this study was $1.019 \pm 0.126$ (the ratio of mean postoperative CDVA to mean preoperative CDVA).

Predictability of the procedure was shown with the help of scattergram, which compared intended SEQ to achieve SEQ at 12 months (Figure 1D). All the points representing achieved SEQ were observed to be present within \pm 1D range of the intended SEQ, shown as the purple line. Graph shows that 207 out of 208 eyes achieved SEQ within \pm 0.50 $\mathrm{D}$ of their intended SEQ.

Accuracy profile is shown in Figure 1E. All the study participants achieved spherical equivalent value within $\pm 1 D$ range (100\%). Around 99.5\% (207 out of 208) eyes had postoperative spherical equivalent (SEQ) within $\pm 0.50 \mathrm{D}$.

To observe the stability of procedure, mean SEQ was plotted at different time points; that is preoperatively, 1month, 6 months and 12 months postoperatively (Figure 1F). It showed significant change from preoperative to postoperative 1 month period. Later, it essentially remained stable and only $3 \%$ ( 6 out of 208) patients showed change of more than $0.5 \mathrm{D}$ during 1 month to 12 months postoperative time period.

Pre- and post-operative comparisons of astigmatism are shown in graph Figure 1G. At 12 months, 96.6\% (201 out of 208) patients had astigmatism less than $0.50 \mathrm{D}$, and $100 \%$ of patients' post-op. astigmatism was within $1.0 \mathrm{D}$ range.

A scatter plot in Figure $1 \mathrm{H}$ compares the target-induced astigmatism (TIA) to surgically-induced astigmatism (SIA) at 12 months postoperatively. Figure 1 l represents the plotting of refractive astigmatism angle of error between TIA and SIA. Only 1 patient's result was outside the $\pm 1.0 \mathrm{D}$ line, rest of all 207 patients were within $\pm 1.0 \mathrm{D}$ range of TIA. The graph shows that spherical equivalent at 12 months of $97.6 \%$ (203) patients was within $\pm 0.50 \mathrm{D}$ range. The percentage of patients having angle of error within \pm 5 degrees was $94.7 \%$ (197), and those having angle of error within \pm 15 degrees was $98 \%$ (204).

\section{DISCUSSION}

The main focus of this study was on presenting the refractive outcomes in the forms of standard graphs designed for publication of results of refractive surgery. ${ }^{9}$ In this study, $97 \%$ of patients achieved 20/25 visual acuity or better at one year. A similar study by Yildrim et al., which originally compared refractive outcomes of mechanical and trans-epithelial PRK has shown that in its mechanically removed epithelial group (comparable to this study group), $80 \%$ of study participants achieved post-op. visual acuity equal to or better than 20/25 at the end of one year. ${ }^{10}$ 
A study carried out mainly on ethnic Chinese males in Singapore reports efficacy index of $0.98,{ }^{11}$ which is comparable to this study group, which observed efficacy index of $1.009 \pm$ 0.132 .

One of the studies by Costa et al., in which PRK was performed using Wavelight Allegretto Wave Eye-Q $®$ Excimer laser system (Wavelight Laser Technologie AG, Erlangen, Germany), yielded that efficacy index was 1.00. The UDVA was better than $20 / 20$ in $86.6 \%$ patients and better than $20 / 25$ in $96.6 \%$ patients. ${ }^{12}$ Their results are comparable to this study results. Another study by Sia et al. reported that in wavefront optimized profile of PRK, $98 \%$ of eyes achieved $20 / 20$ or better vision at six months. ${ }^{13}$

The safety index of this study, at the end of one year, is $1.019 \pm 0.126$, which is comparable to other studies on PRK. Singapore Armed Forces study achieved a safety index of 1.09 at one year, whereas, study by Costa et al. projects the safety index of 1.03 at six months follow-up. ${ }^{12,13}$

In this study, all the participants (100\%) achieved spherical equivalent within the \pm 1D target range of intended SEQ. Sachdev et al. compared PRK to PRK plus cross-linking. In the conventional PRK, $0.5 \mathrm{D}$ of predictability was achieved by $97.4 \% .{ }^{14}$ Whereas, in this study $99.5 \%$ patients were within this range. Another study carried out in Pakistan by Hashmani et al. showed predictability to be $64.9 \% .{ }^{15}$ The marked difference between the results given by Hashmani et al. and this study can be attributed to the difference in the follow-up period. Hashmani et al. compiled their study results at one month; whereas, this study calculated the predictability value at one year postoperatively.

A study by Shapira et al. studied the effect of two nomograms for wavefront optimized PRK on myopia and myopic astigmatism. They recorded that astigmatism of $<0.5 \mathrm{D}$ was achieved in $89.4 \%$ patients with improved nomogram. Whereas, $96.6 \%$ of this study patients reached this landmark. ${ }^{16}$

This study, however, had limitations of shorter duration of follow-up, that is one year only; and observing higher order aberrations and quality of life, remains necessary to further study the effect of this procedure on a patient's day-to-day life.

\section{CONCLUSION}

PRK treatment procedure to be effective, predictable and safe for correction of myopia and myopic astigmatism.

\section{FINANCIAL DISCLOSURES:}

Authors of this article have no financial or proprietary interest in any materials or methods mentioned.

\section{ETHICAL APPROVAL:}

This study was carried out after obtaining approval from the
Institutional Review Board and Ethical Committee of Armed Forces Institute of Ophthalmology.

\section{PATIENTS' CONSENT:}

Informed consents were taken from all patients who participated in the study.

\section{CONFLICT OF INTEREST:}

The authors declared no conflict of interest.

\section{AUTHORS' CONTRIBUTION:}

AF: Drafting of the work.

$\mathrm{SH}$ : Conception and design of the work.

MI: Final approval of the manuscript.

SA: Data acquisition.

SFM: Interpretation of data.

QH: Data analysis.

\section{REFERENCES}

1. Somani SN, Moshirfar M, Patel BC. Photorefractive keratectomy. 2020 Jun 26. In: Stat pearls. Treasure Island (FL): Stat pearls publishing 2021; PMID: 31751077.

2. Radkar PP, Mallikarjun MH. Comparison of patient comfort in alcohol assisted versus mechanical debridement in photorefractive keratectomy (PRK). Ophthalmol Res 2019; 2(1):1-9.

3. Zarei-Ghanavati S, Shandiz JH, Abrishami M, Karimpour M. Comparison of mechanical debridement and trans-epithelial myopic photorefractive keratectomy: A contralateral eye study. J Curr Ophthalmol 2019; 31(2): 135-41. doi: 10.1016/j.joco.2019.01.003.

4. Ripa M, Betts B, Dhaliwal S, Wang K, Pouly S, Chen D, et al. Survey of postoperative pain in photorefractive keratectomy using topical versus oral nonsteroidal anti-inflammatory drugs. Clin Ophthalmol 2020; 14:1459-66. doi: 10.2147/ OPTH.S255441.

5. Chang YM, Liang CM, Weng $\mathrm{TH}$, Chien $\mathrm{KH}$, Lee $\mathrm{CH}$. Mitomycin $\mathrm{C}$ for the prevention of corneal haze in photorefractive keratectomy: A meta-analysis and trial sequential analysis. Acta Ophthalmol 2021; 99(6):652-62. doi: 10.1111/aos.14704.

6. Movahedan H, Namvar E, Farvardin M. Outcomes of photorefractive keratectomy in patients with atypical topography. Electron Physician 2017; 9(11):5684-8. doi: 10.19082/5684.

7. Shehadeh MM, Akkawi MT, Aghbar AA, Musmar MT, Khabbas $M N$, Kharouf MF, et al. Outcomes of wavefront-optimized laser-assisted in-situ keratomileusis and photorefractive keratectomy for correction of myopia and myopic astigmatism over one year follow-up. Open Ophthalmol J 2018; 12:256-63. doi: 10.2174/1874 364101812010256.

8. Mohammadi SF, Alinia C, Tavakkoli M, Lashay A, Chams H. Refractive surgery: The most cost-saving technique in refractive errors correction. Int J Ophthalmol 2018; 11(6):1013-9. doi: 10.18240/ijo.2018.06.20.

9. Dupps WJ Jr, Kohnen T, Mamalis N, Rosen ES, Koch DD, Obstbaum SA, et al. Standardised graphs and terms for refractive surgery results. J Cataract Refract Surg 2011 Jan; 37(1):1-3. doi: 10.1016/j.jcrs.2010.11.010.

10. Yildirim Y, Olcucu O, Alagoz N, Agca A, Karakucuk Y, 
Demirok A. Comparison of visual and refractive results after transepithelial and mechanical photorefractive keratectomy in myopia. Int Ophthalmol 2018; 38(2): 627-33. doi: 10.1007/s10792-017-0501-y.

11. Ang BCH, Yap SC, Toh ZH, Lim EWL, Tan MMH, Nah GKM, et al. Refractive outcomes, corneal haze and endothelial cell loss after myopic photorefractive keratectomy in an Asian population: The Singapore Armed Forces' experience. Clin Exp Ophthalmol 2020; 48(5):558-68. doi: 10.1111/ceo. 13759.

12. Costa E, Franqueira N, Rosa AM, Tavares C, Quadrado MJ, Lobo $C$, et al. Photorefractive keratectomy for myopia and myopic astigmatism correction using the wave light allegretto wave eye-q excimer laser system. Int Ophthalmol 2014; 34(3):477-84. doi: 10.1007/s10792-013-9833-4.

13. Sia RK, Ryan DS, Stutzman RD, Pasternak JF, Eaddy JB, Logan LA, et al. Wavefront-guided versus wavefront-optimized photorefractive keratectomy: Clinical outcomes and patient satisfaction. J Cataract Refract Surg 2015; 41(10): 2152-64. doi: 10.1016/j.jcrs.2015.10.054.

14. Sachdev GS, Ramamurthy S, Dandapani R. Comparative analysis of safety and efficacy of photorefractive keratectomy versus photorefractive keratectomy combined with crosslinking. Clin Ophthalmol 2018; 12:783-90. doi: 10. 2147/OPTH.S156500.

15. Hashmani N, Sharif Hashmani PR, Rajani H, Ahmed J, Kumar J, Kumar A, et al. A comparison of visual outcomes and patient satisfaction between photorefractive keratectomy and femtosecond laser-assisted in situ keratomileusis. Cureus 2017; 9(9):1641. doi: 10.7759/cureus.1641.

16. Shapira Y, Vainer I, Mimouni M, Sela T, Munzer G, Kaiserman I. Myopia and myopic astigmatism photorefractive keratectomy: Applying an advanced multiple regression-derived nomogram. Graefes Arch Clin Exp Ophthalmol 2019; 257(1):225-32. doi: 10.1007/ s00417-018-4101-y. 